\title{
ROSAT HRI OBSERVATIONS OF THE YOUNG STARBURST GALAXY NGC 5253
}

\author{
D. K. STRICKLAND AND I. R. STEVENS \\ School of Physics \& Astronomy, University of Birmingham, \\ Edgbaston, Birmingham, B15 2TT, U.K.
}

\begin{abstract}
Large numbers of Wolf-Rayet stars in the nearby $(4.1 \mathrm{Mpc})$ starbursting dwarf galaxy NGC 5253 indicate the starburst is only $\sim 5$ million years old (Schaerer et al. 1997). This makes NGC 5253 an ideal object for studying the early phases of starburst activity, in particular superbubbles blown by supernovae and winds from massive stars.

Previous low resolution observations suggested the observed X-ray emission was due to a superbubble. We use the enhanced resolution available with a long ROSAT High Resolution Imager (HRI) observation and find that the X-ray emission arises from several unresolved sources associated with the young star clusters seen in the optical. We discuss the possible origins of the point-like emission, and find that they could be small superbubbles blown by the individual clusters of massive stars.
\end{abstract}

\section{Introduction}

Starbursts in dwarf galaxies are predicted to have profound implications for the future of the host galaxy, potentially leading to its destruction. Thermalised SN and winds from the massive stars created in the starburst event form a hot $\left(10^{7}-10^{8} \mathrm{~K}\right)$, X-ray emitting superbubble of metal enriched gas, which eventually blows out of the galaxy as a galactic wind.

In a dwarf galaxy such as NGC 5253 these superbubbles have enough energy to unbind the entire ISM on a timescale of $\sim 10^{7} \mathrm{yr}$, given the shallow gravitational potential. This is one of the mechanisms invoked to explain the disappearance of the excess faint blue galaxies (c.f. De Young \& Heckman 1994). The loss of the heavy elements synthesised in the massive stars will have strong implications for the chemical evolution of these systems. 
The observational existence of full scale galactic winds in the halos of nearby starburst galaxies is well established. These are generally seen in larger spirals rather than dwarfs, and represent the mature galactic wind phase rather than the earlier superbubble phase. NGC 5253 represents a chance to study the earlier superbubble phase in a nearby dwarf galaxy.

\section{X-ray observations of NGC 5253}

A ROSAT PSPC observation of NGC 5253 by Martin \& Kennicutt (1995) found a marginally extended $\left(R \sim 10^{\prime \prime}\right)$ source. From the observed temperature, $T \approx 4 \times 10^{6} \mathrm{~K}$, and luminosity $L_{0.1-2.4 \mathrm{keV}}=\left(6.5_{-1.6}^{+5.8}\right) \times 10^{38} \mathrm{erg} \mathrm{s}^{-1}$ Martin \& Kennicutt concluded that the emission was most likely from a very luminous superbubble, rather than from X-ray binaries or supernova remnants.

In order to constrain the size and morphology of the X-ray emission from NGC 5253, we obtained a $70 \mathrm{ks}$ observation with the ROSAT HRI in July 1996. The HRI has a resolution of $5^{\prime \prime}$, corresponding to $100 \mathrm{pc}$ at the distance of NGC 5253 .

We find that the X-ray emission comes from (at least) five point-like sources, with no obvious bubble-like structure evident. Using the PSPC spectral model to convert count rates to fluxes yields individual $0.1-2.4 \mathrm{keV}$ luminosities in the range $2-8 \times 10^{37} \mathrm{erg} \mathrm{s}^{-1}$.

\section{Discussion}

The similarity in morphology between the X-ray emission and the star clusters seen in the optical (Gorjian 1996, Calzetti et al.1997) suggests that the emission is associated with the young massive stars in the core of nucleus of NGC 5253. The spectral properties, association with young stars and lack of non-thermal radio emission (Beck et al.1996) argues against the point-like X-ray emission being due to Low Mass X-ray Binaries or SN. A set of young, $t \sim 5 \mathrm{Myr}$, superbubbles, with realistic mass and energy injection rates given the observed cluster masses, can provide the required soft X-ray luminosity provided the ambient density is $n_{0} \gtrsim 100 \mathrm{~cm}^{-3}$.

\section{References}

Beck, S. C., Turner J. L., Ho P. T., Lacy J. H., Kelly D. M., 1996, ApJ, 457, 610

Calzetti, D. et al., 1997, ApJ, in press

De Young, D. S., Heckman T. M., 1994, ApJ, 431, 598

Gorjian, V., 1996, AJ, 112, 1886

Martin, C. L., Kennicutt R. C., 1995, ApJ, 447, 171

Schaerer, D., Contini, T., Kunth, D., Meynet G., 1997, ApJ, 481, L75 